\title{
Integrando um Agente Pedagógico para Recomendação de Tutores a um Sistema de Gerência de Cursos
}

\author{
João Pedro Accorsi Ribeiro \\ Eliseo Reategui \\ Elisa Boff
}

\author{
Departamento de Informática - Universidade de Caxias do Sul (UCS) \\ Rua Francisco Getúlio Vargas, 1130 - 95070-560 Caxias do Sul - RS \\ peto@lotinet.com.br, ebreateg@ucs.br, eboff@ucs.br
}

\begin{abstract}
Resumo. A união de novos paradigmas de educação com tecnologia inovadora têm nos apresentado inúmeras ferramentas para aprimorar o ensino a distância e presencial. Os agentes pedagógicos podem humanizar as interfaces destas ferramentas e melhorar a percepção do aluno em relação ao conteúdo apresentado. $O$ objetivo deste trabalho é introduzir um agente pedagógico a um sistema de gerência de cursos. O papel do agente pedagógico proposto é de introduzir um componente sócio-afetivo à interface do sistema e promover a interação entre os alunos. Através do monitoramento das ações do estudante que busca realizar alguma tarefa, o agente é capaz de identificar outros alunos que podem auxiliar este estudante na execução do seu trabalho.
\end{abstract}

Palavras-chave: agentes pedagógicos, sistemas de gerência de cursos, modelo de aluno, recomendação de tutores, aprendizagem colaborativa.

\section{Integrating a Pedagogical Agent for Tutor Recommendation in a Course Management System}

\begin{abstract}
The union of new educational paradigms and cutting edge technology has introduced many tools to enhance distance and traditional learning paradigms. Pedagogical agents may humanize the interfaces of these tools and improve the perception of the students in relation to the contents presented. The main goal of this work is to introduce a pedagogical agent into a course management system. The role of the pedagogical agent proposed here has been to introduce a social-affective component in the system interface and to promote the interaction among students. Through the monitoring of the actions of the users in the system, the agent is capable to identify students who can help others in the execution of certain tasks.
\end{abstract}

Keywords: pedagogical agents, course management sytems, student modelling, tutor recommendation, collaborative learning.

\section{Introdução}

A popularização da Internet trouxe diversas ferramentas para variados setores da sociedade. Um destes setores que continua a se desenvolver é o da educação (Peraya, 1994). Seus 
conceitos estão sendo reavaliados, suas metodologias estão sendo aprimoradas e seu livre acesso é cada vez mais possível. Um dos suportes desta mudança é a implementação de sistemas de Ensino a Distância por diversas entidades do país e do mundo. Muitos desses sistemas são distribuídos sob a licença de software livre, permitindo que seu uso pedagógico e acadêmico seja intensificado. Dentre esta gama de sistemas destacamos os CMS - Course Management Systems - que permitem o total controle sobre o funcionamento de um curso a distância. Rooda, WebCT, AulaNet, Teleduc e Moodle são alguns exemplos de CMSs. Comunidades e grupos de discussão mostram que colaborar é possível, e buscar ajuda ou recursos num espírito de aprendizagem cooperativa é o próximo passo da evolução (Gokhale, 1995).

É justamente nesta realidade que este projeto se enquadra: utilizar e intensificar a colaboração dentro de um contexto de ensino a distância. Mais precisamente na busca pelo elo entre indivíduos de um mesmo grupo, com qualidades e características complementares. Este projeto visa criar um sistema de recomendação de tutores e integrá-lo a um agente pedagógico já existente (Reategui et al., 2005), o qual é capaz de interagir com o usuário em linguagem natural num ambiente interativo de aprendizagem.

O uso de agentes pedagógicos tem se mostrado bastante eficaz nos processos de aprendizagem (Pandzic et. al. 1999). Propomos aqui a integração de um agente pedagógico a um sistema de gerência de cursos para promover a interação entre alunos.

Um modelo de aluno específico foi definido no projeto para considerar nele dados sócioafetivos. Estes modelos são representações que nos detalham aspectos comportamentais, demográficos, afetivos e relacionados ao desempenho (performance) do aluno num determinado domínio (Jeremie e Devedzi, 2004). Com a utilização destes modelos, é possível personalizar a comunicação do agente de interface com o aluno, fornecendo a este um ambiente mais voltado aos seus interesses e necessidades (Sison e Masamichi, 1998).

A próxima seção descreve os agentes de interface, apresentando o estado da arte na área. Em seguida, um modelo de aluno é proposto, sendo utilizado em um algoritmo para recomendação de tutores. A seção 5 do artigo descreve alguns detalhes de implementação do sistema, e a última seção apresenta conclusões e direções para trabalhos futuros.

\section{Agentes de Interface}

O objetivo da área de agentes de interface é desenvolver agentes que possam melhorar o processo de interação entre homem e máquina. Os agentes de interface que possuem uma representação gráfica são chamados de personagens virtuais ou assistentes virtuais, atuando como assistentes que auxiliam o usuário na realização de tarefas num determinado sistema.

Podemos classificar os personagens virtuais segundo suas aplicações, e dentro delas temos as seguintes (Pandzic, 2001):

- Entretenimento: são usados quando o foco do ambiente é a diversão. Geralmente são usadas figuras caricatas de pessoas conhecidas ou outras criaturas divertidas. · Comunicação Pessoal: convites, mensagens, piadas, pedidos, ou qualquer outro tipo de comunicação pessoal pode ser feita através de personagens virtuais. 
- Auxílio de Navegação: são usados para dar boas vindas ao usuário, apresentar uma empresa ou orientar o visitante em relação aos links do website.

- Broadcasting: são usados para, através de apresentadores virtuais, transmitir notícias de acordo com o perfil de usuário.

- Comércio: interagem com os futuros clientes explicando fatos sobre um determinado produto e facilitando a venda e transação por sistemas de e-commerce.

- Educação: são usados pra representar a figura de professores ou instrutores, cumprindo o papel de agentes pedagógicos que fornecem suporte para um ambiente educativo e de ensino à distância.

Um agente de interface é chamado de pedagógico quando atua num sistema aplicado à educação. Ele tem como função principal fazer a comunicação com o aluno, guiando-o nos ambientes e dando suporte às tarefas de aprendizagem. Através deste processo de comunicação e de monitoramento das ações do usuário, o agente é capaz de capturar informações e montar um modelo de aluno. Este modelo é descrito na seção a seguir.

\section{Modelo de Aluno}

Modelo de aluno é definido como a representação do conhecimento e das habilidades cognitivas do aluno num determinado momento (Vicari, 2005). Estas informações contidas no perfil do aluno, podem vir de diversas fontes, como o próprio aluno, a interação do aluno com o ambiente, o professor ou o administrador do sistema.

Além da personalização de conteúdo e do ambiente, os modelos de aluno também são utilizados para reconhecer planos, descobrir caminhos de solução, recomendar conteúdo, analisar performance e desvendar habilidades de resolução de problemas.

Dentre as variadas técnicas de modelagem de aluno, encontramos diversos padrões que são utilizados no mundo, dentre eles:

- $\quad$ Scorm, do Depto. de Defesa dos EUA (Scorm, 2007);

- Eduperson, das empresas Internet2 Middleware e Educause (EDUPERSON, 2007);

- - Ulf, da Saba Software (ULF, 2007);

- · PAPI, da IEEE (IEEE, 2007);

- . IMS LIP, da Global Learning Consortium (IMS LIP, 2007).

Cada um destes modelos possui informações bastante variadas, porém alguns dados são encontrados na maior parte deles, como por exemplo dados pessoais (nome, endereço, idade, sexo), as preferências (língua, interface) e o histórico do aluno (tarefas,atividades, leituras). Estas são algumas das informações que também fazem parte do modelo de aluno utilizado neste trabalho. Além destas, também consideramos algumas variáveis necessárias para a recomendação de tutores, tais como o humor do aluno no momento da utilização do sistema, a performance do aluno em relação aos tópicos em estudo, a aceitação do aluno por outros e o número de interações no ambiente de ensino. 
Tais informações são detalhadas a seguir.

\section{Recomendação de Tutores}

A idéia de recomendação de tutores e a formação de grupos está ligada ao conceito de aprendizado cooperativo. Segundo Gokhale (1995) o termo aprendizado colaborativo se refere a um método de instrução no qual os estudantes de diversos níveis de performance trabalham juntos em pequenos grupos em direção a um objetivo em comum.

Dentro de um ambiente interativo de aprendizagem podem acontecer situações nas quais o material disponibilizado não é suficiente para suprir as necessidades de um aluno.

Nestes casos é importante disponibilizar uma ferramenta de ação humana para que possa haver uma comunicação entre alunos, e que assim, possam construir conhecimento.

Neste contexto, para termos uma recomendação eficiente, são necessários 3 requisitos (Boff et al., 2004):

- Encontrar um estudante capacitado e disposto a ser um tutor;

- Incentivar a criação de um grupo entre o aluno tutor e o aluno em dificuldades;

- - Prover ferramentas para permitir sua comunicação.

Uma das formas de utilizar a teoria da formação de grupos e do estudo colaborativo é desenvolver uma ferramenta que consiga classificar os alunos, de forma a apontar quais, em um determinado momento, estão aptos a serem tutores de alunos com dificuldades.

O algoritmo de recomendação de tutores utilizada neste trabalho é baseado nos atributos e cálculos propostos por (Reategui et al., 2006), e envolve a quantificação de fatores psicológicos e de personalidade em conjunto com o histórico pedagógico do aluno.

O cálculo é feito com a combinação de 4 fatores principais: estado de humor, performance, aceitação e interações.

- Humor: A emoção exerce influência nos processos de raciocínio, e um fator determinante no processo de recomendação de um tutor é saber o estado emocional do aluno em um determinado momento. O seu humor é que vai definir se ele está emocionalmente apto a prover uma orientação efetiva. $\mathrm{O}$ valor do humor utilizado na fórmula é estabelecido pelo próprio aluno, no momento de entrada no sistema de ensino.

- Performance: Representa o desempenho de um aluno num determinado assunto em todos os aspectos propostos, como leituras, tarefas, exercícios e atividades. O cálculo da performance do aluno é feito através da soma de dois grupos, o de leituras que compreende a parte teórica, e o de atividades, que compreende a parte prática ou de exercícios. Dentro de cada grupo, é feito o produto das leituras concluídas com um valor de peso, valor que pode ser definido pelo professor ou pelos administradores do sistema de ensino. A partir deste produto, é feita a divisão do valor obtido pelo total de leituras propostas pelo professor. Na fórmula abaixo podemos analisar como isto é feito:

$$
\mathrm{Pf}=(\mathrm{P} 1 * \mathrm{Lc}) / \mathrm{Lp}+(\mathrm{P} 2 * \mathrm{Ac}) / \mathrm{Ap}
$$


Pf é o escore de performance do aluno num determinado assunto, P1 a constante relativa ao peso que as leituras têm sobre o assunto, Lc o valor das leituras concluídas e Lp o número de leituras propostas pelo professor. Na segunda parte temos P2 representando o peso das atividades, Ac como as atividades concluídas e Ap como as atividades propostas.

- Aceitação: O grau de aceitação nos mostra o quão eficaz é o aluno como tutor. Este valor é correspondente à média das notas que os alunos que solicitaram orientação deram para o aluno tutor ao fim de cada sessão.

- Iterações: É a média das comunicações iniciadas e respondidas pelo aluno. O cálculo é semelhante ao cálculo utilizado na obtenção do fator Performance, como vemos a seguir:

$$
\mathrm{Mi}=(\mathrm{P} 1 * \mathrm{Ci}) / \mathrm{MGi}+(\mathrm{P} 2 * \mathrm{Cr}) \mathrm{MGr}
$$

Sendo Mi a média de iterações do aluno, P1 e P2 representam a importância de cada campo na fórmula, Ci são as comunicações iniciadas, com MGi sendo sua média geral, Cr são as comunicações respondidas, tendo MGr como a média geral.

A partir destes 4 valores, podemos calcular o valor do escore final do aluno. A fórmula final simplesmente atribui um peso a cada atributo. Estes pesos podem variar, podendo amplificar a utilização deste cálculo. De acordo com o objetivo do projeto, um atributo pode ter mais importância que outro. O passo seguinte é a soma dos valores, conforme fórmula abaixo:

$$
\text { Escore }=\mathrm{P} 1 * \mathrm{Eh}+\mathrm{P} 2 * \mathrm{Pf}+\mathrm{P} 3 * \mathrm{Ac}+\mathrm{P} 4 * \mathrm{Mi}
$$

Sendo que Eh corresponde ao estado de humor, Pf equivale ao valor da performance, AC é sua média de aceitação e Mi a média de iterações. Os pesos P1, P2, P3 e P4 correspondem a importância de cada atributo. Esta fórmula tem como objetivo, avaliar a capacidade de um aluno em ser tutor sobre um determinado assunto. Seu escore final determinará se o mesmo está apto ou não a auxiliar um colega.

\section{Implementação}

O sistema gerenciador de cursos Moodle (Moodle, 2007) foi escolhido como plataforma de base para o trabalho. Tal escolha foi fundamentada no fato de que a ferramenta é distribuída sob a licença de software livre e possui uma interface bastante intuitiva, sendo amplamente utilizada no mundo todo.

Com base nos dados dos alunos coletados no log do Moodle, é possível aplicar sobre eles os algoritmos de recomendação de tutores. Duas hipóteses foram criadas para disparar a recomendação: quando já se passaram $\mathrm{X}$ dias desde a última visita do aluno a uma tarefa, e quando o aluno acessou $\mathrm{N}$ vezes uma tarefa porém não a resolveu ainda.

Essas duas variáveis $\mathrm{X}$ e $\mathrm{N}$ podem ser editadas pelo professor ou administrador do sistema, de modo a flexibilizar o momento no qual o agente entra em ação fazendo a recomendação de tutores. $\mathrm{O}$ algoritmo analisa a navegação do aluno pelo ambiente de ensino e, em conjunto com os dados sócio-afetivos coletados, aponta um tutor entre os alunos cadastrados. A interação entre os dois alunos se dá pela ferramenta de chat do próprio Moodle. Com algumas modificações no seu código-fonte, incluímos um mecanismo para 
que o aluno que requisitou o tutoramento possa qualificar o seu tutor, ação fundamental para o correto funcionamento do cálculo de escore proposto neste trabalho.

Além de apresentar aos alunos as recomendações de tutores, o agente pedagógico utilizado, chamado Cadinho, é capaz de dar dicas de navegação e responder a perguntas em linguagem natural. $O$ sistema de comunicação do agente utiliza a linguagem de representação AIML (Artificial Intelligence Markup Language - Linguagem de Marcação de Inteligência Artificial) (Wallace, 2003). Esta linguagem foi desenvolvida e utilizada no desenvolvimento de um dos chatbots mais conhecidos até hoje, chamado A.L.I.C.E. O mecanismo utilizado no desenvolvimento do robô é o de estímuloresposta.

O sistema armazena estímulos (frases e fragmentos que podem ser utilizados para questionar o robô) e os emprega na busca a respostas previamente estabelecidas.

A linguagem descreve classes de objetos chamados de objetos AIML, e descreve parcialmente o comportamento do programa que os processa.

Alguns dos principais marcadores da linguagem são:

<aiml >: tag de início de documento

$<$ categoria >: tag que marca as unidades de conhecimento em uma base

<pattern>: utilizado para conter um padrão que será buscado nos diálogos que os usuários entrarão para se comunicar com o assistente

$<$ template >: contém as respostas às entradas feitas pelo usuário.

Além dos marcadores já existentes para descrever conhecimento em AIML, é possível criar novos marcadores. Cadinho foi descrito utilizando-se os marcadores padrão do AIML, com exceção de um marcador desenvolvido para controlar sua mudança de seu estado. Este marcador, chamado de <humor $>$, foi utilizado para controlar a mudança de imagens que refletem os diferentes estados de espírito do personagem (feliz, receptivo, incomodado, etc.). Deste modo, ao introduzir uma pergunta ao assistente (i.e. um estímulo), o assistente busca em sua base uma resposta apropriada e a apresenta, trocando de estado quando necessário.

O banco de conhecimento desenvolvido para o assistente Cadinho armazena informações relativas a algoritmos, permitindo que o assistente auxilie os alunos principalmente em questões teóricas. Por ter sido desenvolvido com as mesmas tecnologias do Moodle (PHP e MySQL), o agente pedagógico foi facilmente integrado ao sistema gerenciador de cursos. A Figura 1 mostra uma tela do Moodle na qual podemos ver o agente de interface recomendando ao aluno Pedro que interaja com outro aluno também conectado ao sistema. 


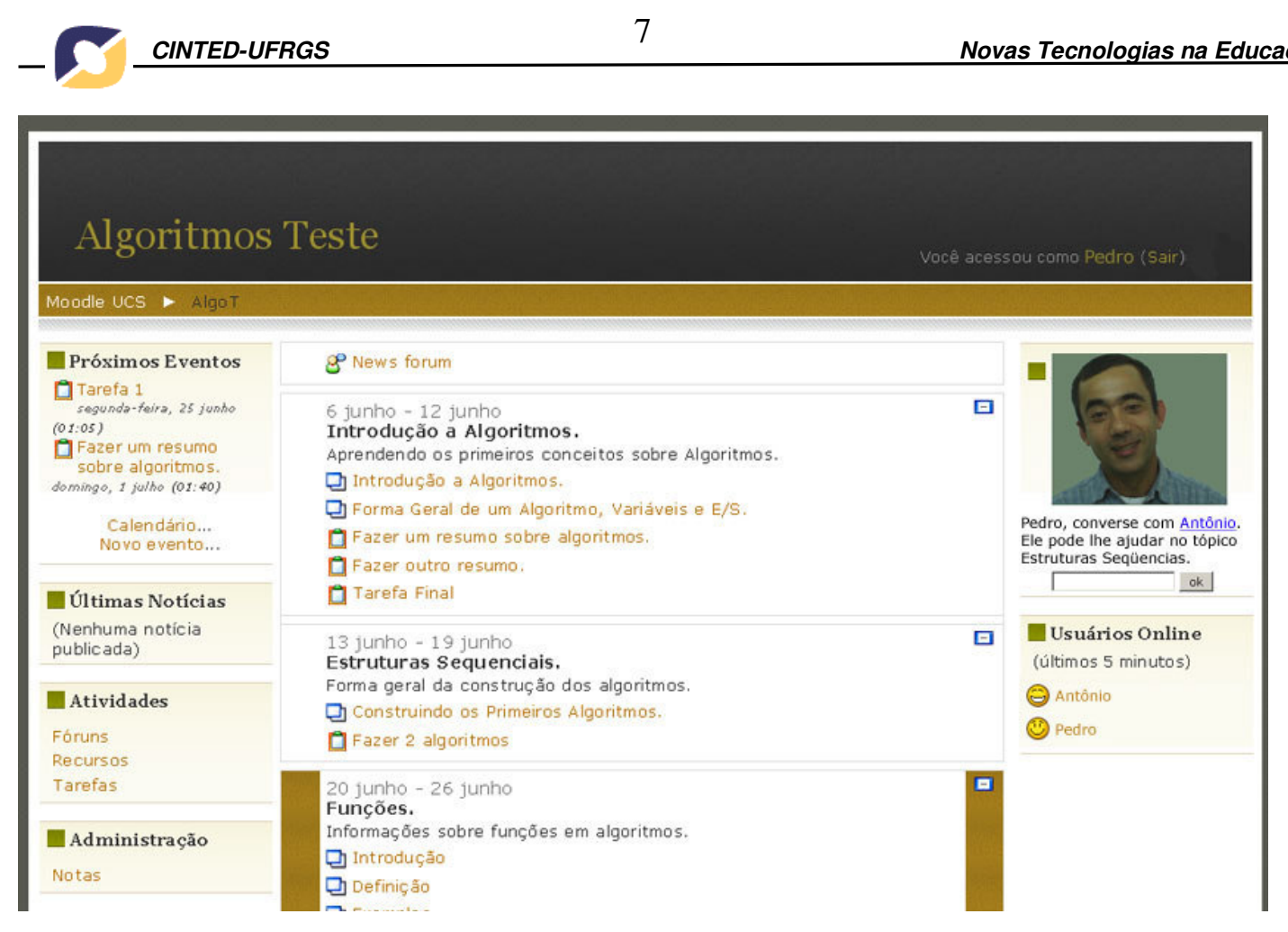

Figura 1 - Agente Pedagógico recomendando tutor no Ambiente Moodle

\section{Considerações Finais}

Os agentes de interface têm sido cada vez mais empregados para criar formas de interação amigáveis com o usuário, introduzindo componentes afetivos que alteram a forma com que o usuário percebe sua comunicação com a máquina. Nos anos 1990, Pattie Maes (1994) já apontava que através dos agentes de interface, o usuário poderia interagir com os sistemas computacionais e ser engajado em um processo cooperativo no qual homem e máquina poderiam iniciar a comunicação um com o outro, monitorar eventos e realizar tarefas. Tal forma de interação era fundamentada na metáfora de um ajudante que colaborava com o usuário no mesmo ambiente de trabalho.

Neste trabalho, foi proposta a introdução de um agente pedagógico ao sistema de gerência de conteúdo Moodle. O agente proposto é capaz de atuar em uma área específica, no caso aprendizagem de algoritmos. Tendo sido implementado através da linguagem de marcação AIML, seria relativamente simples ampliar as capacidades comunicativas do agente para outras áreas, através da expansão do seu banco de dados.

Portanto, o mesmo agente poderia ser aplicado a outras disciplinas, mantendo sua capacidade de recomendação de tutores.

A utilização de um agente pedagógico junto ao Moodle reforça a importância da dimensão sócio-afetiva nos componentes da interface do sistema. Na educação, a ênfase dada a esta dimensão torna-se ainda mais importante, sabendo-se da relevância da afetividade e das trocas sociais nos processos de aprendizagem (Vygotsky, 1987). Lester et al. (1997), por exemplo, mostraram que a presença da figura humana teve um efeito positivo nas experiências interativas dos estudantes. André et al. (1999) confirmaram estas hipóteses em um experimento, mostrando que os estudantes consideraram o assunto em estudo 
significativamente menos difícil e a apresentação mais lúdica na presença de um agente pedagógico. Neste mesmo experimento a maior parte dos estudantes afirmou que o assistente os auxiliou a prestar atenção a informações relevantes.

Além de introduzir estímulos sócio-afetivos, o agente pedagógico empregado neste trabalho, tem a capacidade de recomendar tutores, estimulando a interação entre alunos e promovendo a aprendizagem colaborativa. Tal abordagem é apoiada em teorias que apontam para a importância das interações sociais no processo de aprendizagem

(Vygotsky, 1987).

Apesar de todos os benefícios relacionados ao uso dos agentes animados, sua utilização pode eventualmente perturbar o usuário habituado a uma interface na qual não existe nenhum mediador. Dentro desta perspectiva, o design de um agente de interface possui ainda muitos desafios, tais como decidir qual o nível de controle a ser delegado ao agente e como desenvolver a confiança do usuário no agente. Ambos os desafios podem ser superados através da adoção, por parte do agente, de comportamentos que estejam de acordo com o contexto da interação. Estes comportamentos devem ser projetados pelos pesquisadores e educadores, no caso específico de agentes pedagógicos animados, a fim de que o agente possua ilusão de vida levando os usuários a uma experiência interativa mais interessante e motivadora do processo de aprendizagem.

\section{Referências}

ANDRE, E., RIST, T., E MULLER, J. (1999). Employing AI methods to control the behavior of animated interface agents. Applied Artificial Intelligence, Vol. 13, Num. 4- 5, May, 415-448.

BOFF, Elisa; GOMES, Eduardo Rodrigues; VICARI, Rosa Maria. Social, Affective and Pedagogical Agents for the Recommendation of Student Tutors. In: 7 International Conference on Intelligente Tutoring Systems, 2004, Maceió.

Workshop on Social and Emotional Intelligence in Learning Environments.

EDUPERSON (2007). EduPerson Object Class Specification. Disponível em http://www.nmi-edit.org/eduPerson/internet2-mace-dir-eduPerson-200210.pdf. Junho de 2007.

GOKHALE, Anuradha. (1995). Collaborative Learning Enhances Critical Thinking. Journal of Technology Education Vol. 7 No. 1, Fall 1995.IEEE (2007).

PAPI Learner, Draft 8 Specification. Disponível em http://edutool.com/papi/. Junho de 2007.

IMS LIP (2007). IMS Learner Information Packaging Information Model Specification. Disponível em http://www.imsglobal.org/profiles/lipinfo01.html. Junho de 2007.

JEREMIE, Z. e DEVEDZI, V. (2004). Design Pattern ITS: Student Model Implementation. Proceedings of the IEEE International Conference on Advanced Learning Technologies.

LESTER, J. C., CONVERSE, S. A., KAHLER, S. E., BARLOW, S. T., STONE, B. A. and BHOGAL, R. S. The Persona Effect: Affective Impact of Animated Pedagogical Agents. Proceedings of ACM CHI 97 - Human Factors in Computing Systems. Atlanta, GA, USA. 1997.

MAES, P. (1994). Agents that reduce work and information overload. Communications of the ACM, 37(7):31-40.

MOODLE (2007). Website do CMS Moodle. Disponível em http://www.moodle.org. Junho de 2007. 
PANDZIC, I. S., OSTERMANN, J. and MILLEN, D. (1999). User Evaluation: Synthetic Talking Faces for Interactive Services. The Visual Computer, Springer Verlag, vol. 15. pp. 330-340.

PANDZIC, I. S. (2001). Life on the Web. Software Focus Journal. Wiley, NY, 52-58. PERAYA, D. (1994). Distance Education and de WWW. Disponível em: http://tecfa.unige.ch/edu-comp/edu-ws94/contrib/peraya.fm.html. Capturado em Dezembro 2006: REATEGUI, E.; BOFF, E.; LORENZATTI, A. Proposta e Avaliação Preliminar de um Assistente Virtual para Recomendação de Conteúdos. In: SIMPÓSIO BRASILEIRO DE INFORMÁTICA NA EDUCAÇÃ̂, Juiz de Fora. Proceedings XVI Simpósio Brasileiro de Informática na Educação. SBC, 2005.

REATEGUI, Eliseo; BOFF, Elisa; CERON, Rafael Fernando; VICCARI, Rosa Maria. Um Agente Animado Sócio-Afetivo para Ambientes de Aprendizagem. Revista Brasileira de Informática na Educação - Edição Especial Aprendizagem Colaborativa. v. 14 n.3. Setembro-Dezembro de 2006. p27-38.

SCORM (2007). Scorm Overview. Disponível em http://xml.coverpages.org/SCORM- 12Overview.pdf. Junho de 2007.

SISON, R. e MASAMICHI, S. (1998). Student Modeling and Machine Learning. International Journal of Artificial Intelligence in Education, 9, 128-158.

ULF (2007). Universal Learning Format. Technical Specification. Disponível em http://xml.coverpages.org/ulfSpecification20001204.pdf. Junho de 2007.

VICARI, R. Delineamento de um Agente para detectar a Auto-Eficácia do Aluno no Ensino-Aprendizagem via Web. In: Novas Tecnologias na Educação, CINTED-UFRGS.

2005.

VYGOTSKY, L. S. The collected works of L. S. Vygotsky. New York: Plenum Press, c1987-c1999 v. 1-6.

WALLACE, R. (2003). "The Elements of AIML Style", ALICE A. I. Foundation 